\title{
A mathematical model for surface roughness of fluidic channels produced by grinding aided electrochemical discharge machining (G-ECDM)
}

\author{
Ladeesh V. G. ${ }^{1 \mathrm{a}}$ and Manu R. \\ ${ }^{1}$ Dept. of Mechanical Engineering, National Institute of Technology Calicut, Calicut, Kerala-673 601
}

\begin{abstract}
Grinding aided electrochemical discharge machining is a hybrid technique, which combines the grinding action of an abrasive tool and thermal effects of electrochemical discharges to remove material from the workpiece for producing complex contours. The present study focuses on developing fluidic channels on borosilicate glass using G-ECDM and attempts to develop a mathematical model for surface roughness of the machined channel. Preliminary experiments are conducted to study the effect of machining parameters on surface roughness. Voltage, duty factor, frequency and tool feed rate are identified as the significant factors for controlling surface roughness of the channels produced by G-ECDM. A mathematical model was developed for surface roughness by considering the grinding action and thermal effects of electrochemical discharges in material removal. Experiments are conducted to validate the model and the results obtained are in good agreement with that predicted by the model.
\end{abstract}

\section{Introduction}

Machining of ceramics and composites are gaining more importance in the modern industries due to its wide spread application in various fields. Development of new energy efficient and accurate techniques for ceramic machining is a challenging task for scientific community. Grinding aided electrochemical discharge machining is a promising technology, which can be effectively utilized for machining brittle materials like glass and advanced ceramics. A G-ECDM machine consist of a diamond coated solid engraving tool maintained as cathode and an auxiliary electrode kept at a distance of 4-6cm from the tool as anode. Both the electrodes will be dipped in an electrolyte. When a direct current (DC) is applied across the electrodes, hydrogen bubbles will be liberated due to electrochemical reaction. These bubbles act like a series of switches connected to the circuit. The current flows from the tool electrode to the electrolyte through the interface between bubbles called bubble bridges. Due to sudden ohmic heating, some of the bubble bridges blow off, thereby opening the circuit quickly. This switching-off action [1] causes a sudden fluctuation in current thereby inducing an e.m.f. This will cause ionization of the gas inside the bubble and a discharge will be produced. The cycle repeats several thousands of times per second. When a workpiece is placed in the vicinity of these discharges, material removal takes place by melting. Thus the material beneath the tool will be softened by the discharges and the rotating diamond coated tool will remove the material

\footnotetext{
${ }^{\mathrm{a}}$ Corresponding author : ladeesh.vg@gmail.com
} 
from the machining zone by grinding action. A portion of the material will be removed by chemical etching action in which the molten workpiece material reacts with the electrolyte to form complex compounds. These combined mechanisms enhance the material removal rate and the grinding action of diamond grits remove the recast layer produced by solidification of molten material.

Basak and Ghosh [1] experimentally found that the inductance of the circuit plays a predominant role in controlling material removal rate (MRR). An enhancement in MRR was observed with an extra inductance in the circuit. They developed a mathematical model to predict MRR in electrochemical discharge machining of glass. Chak and Rao [2] performed trepanning of alumina by ECDM using an abrasive tool. He observed that the use of pulsed DC reduced the cracking at high voltage and machining ability got improved with the abrasive tool. Jain et al. [3] performed electrochemical discharge machining of alumina and glass using conventional cutting tool (CCT) and abrasive cutting tool (ACT). The material removed for different voltages and temperatures was found to be high for ACT when compared with CCT. Ladeesh and Manu [4] performed drilling of borosilicate glass using G-ECDM and observed that thermal melting, grinding action and chemical etching are the different mechanisms contributing material removal. Liu et al. [5] used grinding aided ECDM for the machining of composites (alumina particles reinforced aluminium alloy) for the first time. In most of the studies mentioned above, researchers tried to improve the process of drilling using ECDM with the help of abrassive tools. No previous work has been reported on using abrasive tool for machining channels or slots on ceramics with the aid of ECDM.

In the present study an attempt has been made to develop fluidic channels on glass using the technique of G-ECDM. The significant machining parameters are investigated with the help of systematic experiments. A mathematical model is developed to predict the surface roughness of the channels produced using G-ECDM.

\section{Experimental methodology}

\subsection{Experimental setup}

A CNC router machine was used to facilitate the motion of the tool in $\mathrm{X}, \mathrm{Y}, \mathrm{Z}$ direction which is shown in Fig. 1(a). The tool used is a diamond coated solid engraving tool of diameter $3 \mathrm{~mm}$, which is shown in Fig. 1(b). A stainless steel plate of dimension $200 \mathrm{~mm} \times 10 \mathrm{~mm} \times 0.7 \mathrm{~mm}$ was used as the auxiliary electrode maintained as anode. The electrolyte used was potassium hydroxide. A customized DC power supply with provision for varying frequency and duty ratio was used for the experiments. Workpiece used was a borosilicate glass of dimension $200 \mathrm{~mm} \times 50 \mathrm{~mm} \times 5 \mathrm{~mm}$

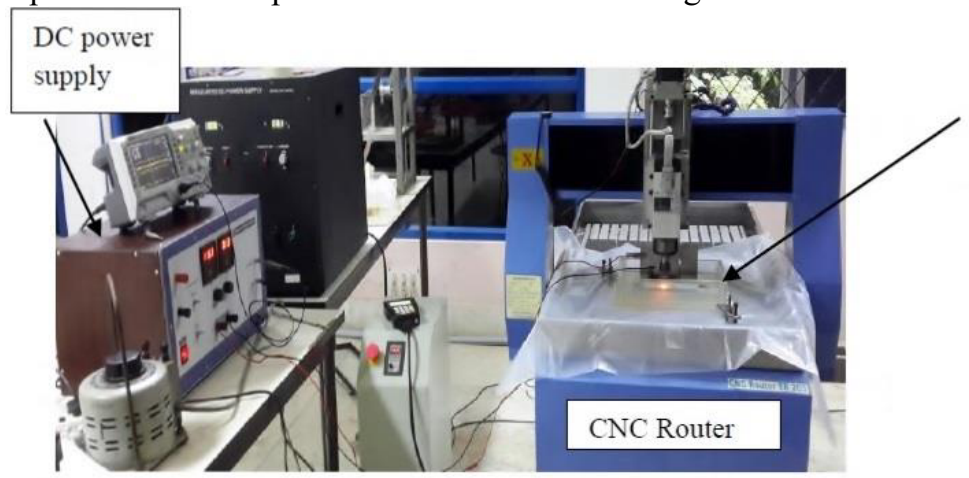

(a)



Machining chamber

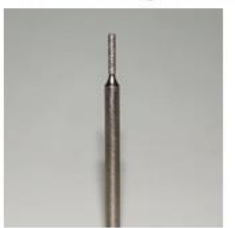

(b)

Figure 1. (a) Machining setup (b) diamond core engraving drill

\subsection{Design of experiments}


An experiment was conducted using Plackett-Burman design. This design is widely accepted for conducting preliminary experiments. The design is more efficient and economical when compared to two level full factorial designs. In the present study, six factors are selected which includes tool rotational speed, feed rate, voltage, duty factor, frequency and electrolyte concentration. Each factor has two levels, which makes a total of 12 experimental runs. Two replications are taken. The selected factors and their levels are given in Table 1. The Placket-Burman design layout used for developing experimental runs are shown in Table 2. Surface roughness of the machined channels are measured using a contact type surface roughness tester (Mitutoyo- Surftest SJ-210). From the main effects plot shown in Fig. 2., it is evident that the Ra value reduces with increase in feed, increase in frequency, reduction of voltage and reduction of duty factor. Analysis of variance (ANOVA) was conducted using a software MINITAB-17 to identify the significant factors. Results of ANOVA are given in Table 3. From the F-test, factors with high F-values like voltage, duty factor, frequency and feed rate are found to be significant and the effect of concentration and tool rotation speed are found to be insignificant.

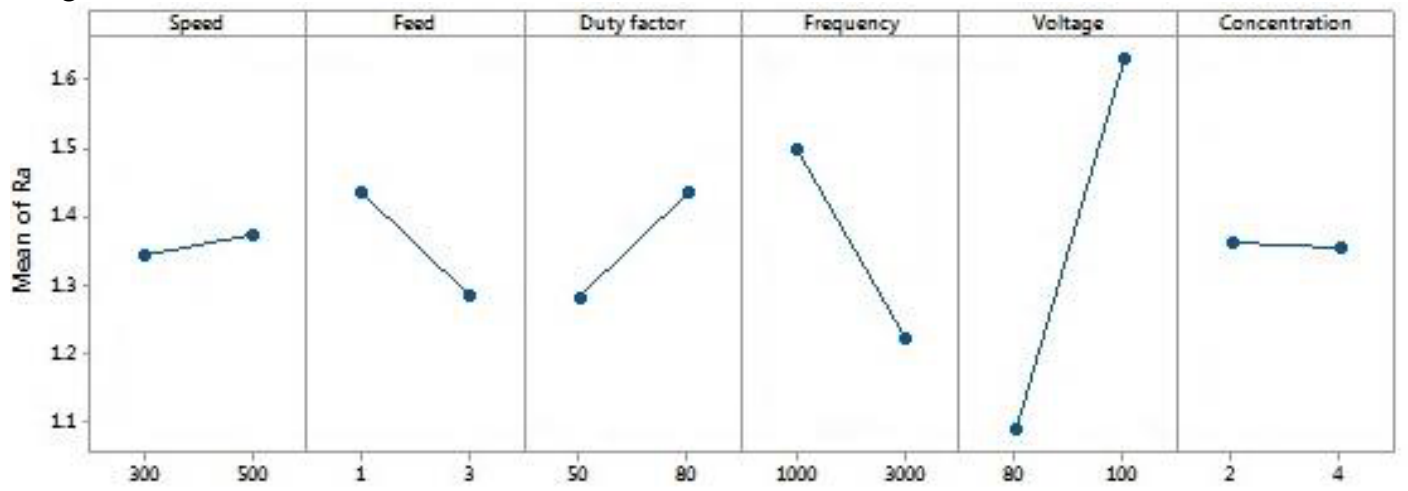

Figure 2. Main effects plot for Surface Roughness (Ra)

Table 1. Factors and levels

\begin{tabular}{|c|c|c|c|}
\hline Factors \& & & \multicolumn{2}{|c|}{ Levels } \\
\cline { 2 - 4 } Notation & Unit & $\begin{array}{r}\text { Low } \\
\mathbf{( - 1 )}\end{array}$ & $\begin{array}{c}\text { High } \\
(+1)\end{array}$ \\
\hline Speed (A) & $\mathrm{rpm}$ & 300 & 500 \\
\hline Feed (B) & $\mathrm{mm} / \mathrm{min}$ & 1 & 3 \\
\hline Duty factor (C) & $\%$ & 50 & 80 \\
\hline Frequency (D) & $\mathrm{Hz}$ & 1000 & 3000 \\
\hline $\begin{array}{c}\text { Voltage (E) } \\
\text { Concentration } \\
\text { (F) }\end{array}$ & $\mathrm{V}$ & 80 & 100 \\
\hline \multicolumn{2}{|c}{$\mathrm{M}$} & 2 & 4 \\
\hline
\end{tabular}

Table 2. Plackett-Burman Design

\begin{tabular}{|c|c|c|c|c|c|c|}
\hline Runs & A & B & C & D & E & F \\
\hline 1 & 1 & -1 & 1 & -1 & -1 & -1 \\
\hline 2 & 1 & 1 & -1 & 1 & -1 & -1 \\
\hline 3 & -1 & 1 & 1 & -1 & 1 & -1 \\
\hline 4 & 1 & -1 & 1 & 1 & -1 & 1 \\
\hline 5 & 1 & 1 & -1 & 1 & 1 & -1 \\
\hline 6 & 1 & 1 & 1 & -1 & 1 & 1 \\
\hline 7 & -1 & 1 & 1 & 1 & -1 & 1 \\
\hline 8 & -1 & -1 & 1 & 1 & 1 & -1 \\
\hline 9 & -1 & -1 & -1 & 1 & 1 & 1 \\
\hline 10 & 1 & -1 & -1 & -1 & 1 & 1 \\
\hline 11 & -1 & 1 & -1 & -1 & -1 & 1 \\
\hline 12 & -1 & -1 & -1 & -1 & -1 & -1 \\
\hline
\end{tabular}

Table 3. ANOVA for Ra

\begin{tabular}{|c|c|c|c|c|}
\hline Source & DoF & $\begin{array}{c}\text { Sum of } \\
\text { squares }\end{array}$ & $\begin{array}{c}\text { Mean } \\
\text { squares }\end{array}$ & F-Value \\
\hline A & 1 & 0.0053 & 0.0053 & 0.39 \\
\hline B & 1 & 0.1406 & 0.1406 & 10.48 \\
\hline C & 1 & 0.1437 & 0.1437 & 10.71 \\
\hline D & 1 & 0.4551 & 0.4551 & 33.91 \\
\hline E & 1 & 1.7642 & 1.7642 & 131.46 \\
\hline F & 1 & 0.0004 & 0.0004 & 0.03 \\
\hline Error & 17 & 0.2281 & 0.0134 & \\
\hline Total & 23 & 2.7373 & & \\
\hline
\end{tabular}

*DoF-Degrees of Freedom

\section{Mathematical Modelling}

Three stages can be identified in an ECDM wave form. In stage-I, a gradual rise of voltage happens till the critical voltage $\left(\mathrm{V}_{\mathrm{c}}\right)$ is reached and suddenly the voltage drops to an arc maintaining voltage. In stage II the arc maintaining voltage continues till the pulse-off time. In the third stage a gradual drop in the voltage to zero can be observed. Stage I can be called as the ignition delay in which the chemical action causing hydrogen liberation and gas film formation happens. Hence the major energy available for the spark is given by arc maintaining voltage $\left(\mathrm{V}_{\mathrm{a}}\right)$. For ECDM using voltage greater than 
the critical voltage, ignition delay is almost absent as shown in Fig. 3(b) when compared to normal ECDM wave form shown in Fig. 3(a)and the duration for arcing is more, which means that more energy can be supplied by the discharge. This is because of the drastic rise in the bubble generation rate at very high voltage above critical voltage. This high bubble concentration permits the discharge to initiate immediately soon after the pule is switched on after a discharge cycle thereby reducing the ignition delay period significantly. Thus, the voltage pulses can be simplified and assumed as rectangular pulses with the arc duration $t_{a}$.

\subsection{The model for material removal rate (MRR)}

The following assumptions are made for developing a MRR model for G-ECDM.

- The workpiece is an electrically non conductive material and the material removal takes place mainly by melting due to electrochemical discharges.

- ECDM happens by a series of sparks and each spark contributes for the formation of a crater.

- The crater produced will be in the form of a portion of sphere (spherical cap).

- Applied voltage is well above critical voltage and the Ignition delay is absent in all discharge cycles

- The tool feed rate is very low, so that the grinding action will be only used to flush the material removed by thermal effects of electrochemical discharges.

From Fig. 4, the total cycle time can be expressed as a sum of pulse-on time or arcing period $\left(t_{a}\right)$ and pulse-off time or idle time $\left(t_{i}\right)$ which is given by (1).

$$
T_{c}=t_{a}+t_{i}
$$

Accordingly, the arc energy can be determined using (2).

$$
E_{a}=\int_{0}^{t_{a}} I_{a}(t) V_{a}(t) d t
$$

The energy transferred to the workpiece will be much less than the energy supplied by arcing. The percentage of energy transferred to the workpiece is given by a term called coefficient of energy transfer $\left(C_{T}\right)$ whose value is around 0.2 [6]in the case of ECDM. Hence the total energy transferred to the work can be expressed as (3). The transferred energy will cause the material removal in the form of micro craters.

$$
E_{T}=\int_{0}^{t_{p}} C_{T} I_{a}(t) V_{a}(t) d t
$$

The volume of the crater produced by a single spark is proportional to the transferred energy and the volume of the crater can be determined by (4) and (5)

$$
\begin{aligned}
& W_{c}=\mu \int_{1} \int_{0}^{t_{a}} C_{T} V_{a}(t) I_{a}(t) d t \\
& W_{c}=\mu \int_{0}^{t_{a}} V_{a}(t) I_{a}(t) d t
\end{aligned}
$$

where $\mu_{l}$ is the proportionality constant and $\mu=\mu_{l} C_{T}$.
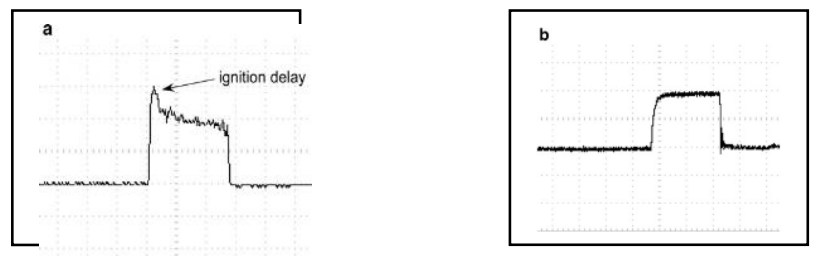

Figure 3. Typical voltage waveform of (a) Normal ECDM voltage wave form (b) ECDM above critical voltage 




Figure 4. Voltage and current waveforms of G-ECDD

Basak et al. [1] found experimentally a relation connecting volume of the material removed per spark and the energy supplied per spark for soda lime glass, which is given by (6)

$$
W_{c}=0.7 \times 10^{-5} E_{a}^{1.5}
$$

If $f$ is the frequency of discharge, the material removal rate can be approximated using the expression (7)

$$
M R R=\delta . W_{c} \cdot f
$$

Where $\delta$ is the density of the workpiece material. Frequency of discharge $f_{s}$ can be calculated as [7]:

$$
f_{s}=\frac{R_{T}}{L} \ln \left[\frac{V_{0}-V_{w}}{V_{0}-V_{c}}\right]
$$

where $R_{T}$ is the total resistance, which is a sum of electrolyte bulk resistance $\left(R_{b}\right)$ and resistance at the interface of tool and electrolyte at the time of discharge $\left(R_{c}\right) . L$ is the circuit inductance, $V_{0}$ is the applied voltage, $V_{w}$ is the water decomposition potential and $V_{c}$ is the critical voltage. In G-ECDM, the major portion of material removal happens by the discharges at the lateral face of the tool. Hence, the spark frequency at the lateral face of the tool $\left(f_{L}\right)$ can be determined by (9)

$$
f_{L}=f_{s} A_{L} / A_{s}
$$

where $A_{s}$ and $A_{L}$ are area of the tool in the electrolyte and area of lateral face of the electrode dipped in the electrolyte. Thus, the equ. (7) for MRR can be modified as:

$$
M R R=0.7 \times 10^{-5} E_{a}^{1.5} \times \delta\left[\frac{A_{L}}{A_{s}} \times \frac{R_{T}}{L} \ln \left(\frac{V_{0}-V_{w}}{V_{0}-V_{c}}\right)\right]
$$

The above equation for MRR is applicable for glass only. A generalized model for MRR is given by $(11)$

$$
M R R=\mu E_{a}^{\lambda} \times \delta\left[\frac{A_{L}}{A_{s}} \times \frac{R_{T}}{L} \ln \left(\frac{V_{0}-V_{w}}{V_{0}-V_{c}}\right)\right]
$$

Where $\mu$ and $\lambda$ are constants, which depends mainly on workpiece material.

\subsection{Model for surface roughness}

In ECDM, the material removal initially happens by melting due to the thermal energy of the spark and small craters will be produced. The removed molten material forms a build-up- edge (BUE) around the crater as shown in Fig. 5(a). The random craters finally overlap and removes material layer by layer. The grinding action of the grits will remove the remaining build-up-edges and finally the peak of the crusts of overlapped craters will be ground by the rotating diamond grits. The final 
finished surface of G-ECDD will appear as shown in Fig. 5(b). The average peak to valley value of surface roughness will be given by the final average peak height of the crusts $(b)$ on finished surface.

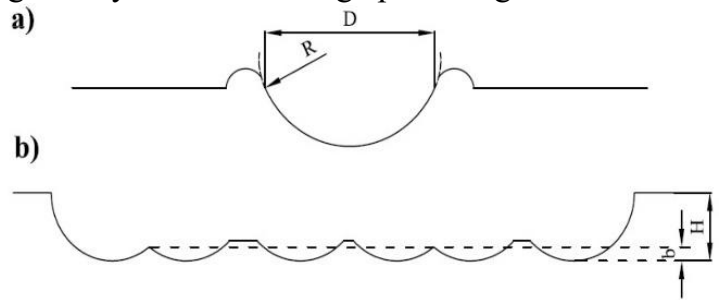

Figure 5. Illustration of the craters (a) single crater with BUE (b) Finished surface produced by G-ECDM.

Volume of the crater produced by a single spark is given by (12)

$$
W_{c}=\frac{1}{3} \pi H^{2}(3 R-H)
$$

Substitute the Eq. (6) on (12), we will get:

$$
0.7 \times 10^{-5} \times E_{a}^{1.5}=\frac{1}{3} \pi H^{2}(3 R-H)
$$

The ratio of the crater depth $(H)$ to the crater diameter $(D)$ for ECDM is in the range 0.2- 0.3 [8]. Assuming that, the average diameter of the crust is approximately equal to the radius of curvature $(R)$ of the spherical crater. Taking H/D ratio as 0.25 and simplifying Eq. (13), we will get the following expression (14) for $H$.

$$
H=8.467 \times 10^{-3} E_{a}^{0.5}
$$

The average peak to valley height $R_{t}$ is given by the average crater height $b$ of the overlapped craters. From microscope observation, the value of $b$ is found to be $1 / k^{\text {th }}$ of $\mathrm{H}$. $k$ depends on the diamond grit size, whose value can be chosen from Table 4. Hence, Rt can be written as:

$$
R_{t}=\frac{1}{k}\left(8.5 \times 10^{-3} E_{a}^{0.5}\right)
$$

Substituting (2) in (15) and simplifying, we get:

$$
R_{t}=\frac{1}{k} 8.5 \times 10^{-3}\left(V_{a} I_{a} t_{p}\right)^{0.5}
$$

Thus, surface roughness during G-ECDM can be expressed as a function of arc maintaining voltage, current and pulse-on period. The surface roughness measurements using contact type method revealed that the centre line average surface roughness value $(R a)$ is $1 / 9$ th of $R_{t}$ value for G-ECDM.

Table 4. Value of $k$ for different grit size

\begin{tabular}{|c|c|c|}
\hline U. S Mesh & In microns & $k$ \\
\hline $60-80$ & $250-177$ & 5 \\
\hline $100-140$ & $149-105$ & 7 \\
\hline $170-230$ & $88-63$ & 9 \\
\hline
\end{tabular}
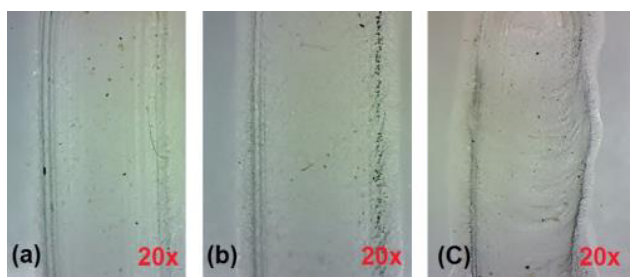

Figure 6. Fluidic channels machined at: a) $80 \mathrm{~V}$, $50 \%, 500 \mathrm{~Hz}, 3 \mathrm{M} \mathrm{b}) 90 \mathrm{~V}, 60 \%, 2 \mathrm{kHz}, 3 \mathrm{M} \mathrm{c})$ $100 \mathrm{~V}, 70 \%, 3 \mathrm{kHz}, 3 \mathrm{M}$

\section{Experimental Validation of the Model}

Experiments are conducted to validate the model for surface roughness. The microscopic images of machined channels are shown in Fig. 6. The same experimental setup used for conducting G-ECDM was used for performing validation experiments. Fig. 7 and Fig. 8 shows the Ra value obtained for 
channels machined using different voltages and different pulse-on time respectively. A low feed rate of $10 \mu \mathrm{m} / \mathrm{s}$ was used so that the effect of feed rate is insignificant.

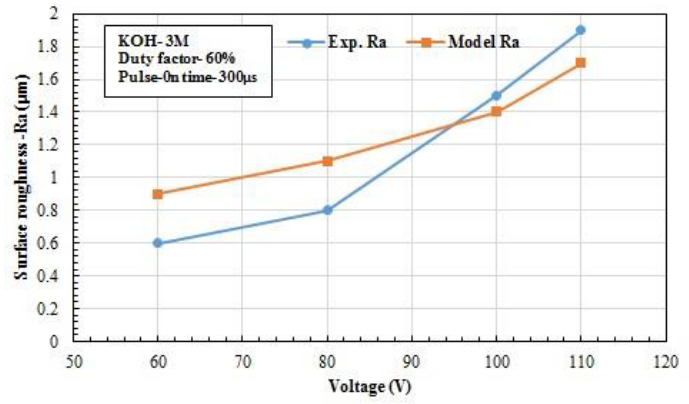

Figure. 7 Effect of voltage on surface roughness

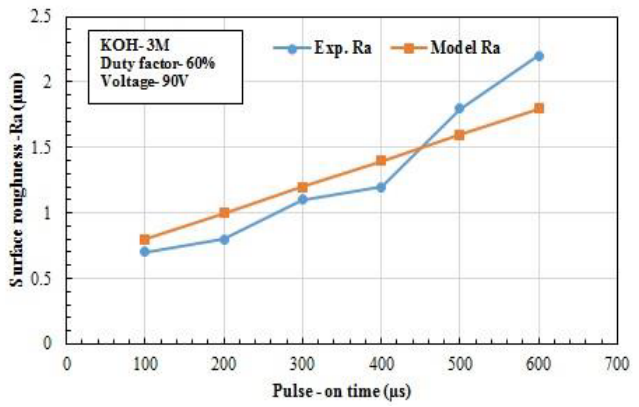

Figure. 8 Effect of pulse-on time on surface roughness

It can be observed from Fig. 7 and Fig. 8, that Ra increases with an increase in voltage and pulse- on time. Discharges at higher voltage produce craters of more depth, which will increase the Ra value. Similarly, a pulse with more time period will produce an arc, which will allow to dissipate the discharge energy for long time. This will increase the depth of the craters and thereby increases the $R a$ value. The obtained experimental values for $\mathrm{Ra}$ are in close agreement with the mathematical model for the channels machined at different voltages. The effect of feed rate will become significant in controlling surface roughness at higher feed values.

\section{Conclusions}

- From the experiment, the significant factors that contributes surface roughness of the channel machined using G-ECDM are identified as voltage, frequency, duty factor and feed rate. The effect of tool rotation speed and electrolyte concentration was found to be insignificant.

- Surface roughness decreases with decrease in voltage, duty factor, feed rate and increase in frequency

- A mathematical model is developed, which can fairly predict the surface roughness of channels machined using G-ECDM. The model correlates arc maintaining voltage, current and pulse-on period with surface roughness.

\section{References}

1. Indrajit Basak, Amitabha Ghosh, J. of Mat. Proc. Tech. 71, 350-359 (1997)

2. S. K. Chak, P. V. Rao, Int. J. of Mach. Tools \& Manuf. 47, 2061-2070 (2007).

3. V. K. Jain, S. K. Choudhury, K. M. Ramesh, Int. J. of Mach. Tools \& Manuf. 42, 1269-1276 (2002).

4. V. G. Ladeesh, R. Manu, Procedia Tech. 25, 1122 - 1128 ( 2016)

5. J. W. Liu, T. M. Yue, Z. N. Guo, Int. J. of Mach. Tools and Manuf. 50, 86-96 (2010).

6. Chenjun Wei, Kaizhou Xu, Jun Ni, Adam John Brzezinski, Dejin Hu, Int. J Adv. Manuf. Tech. 54, 987-995 (2011).

7. Rolf Wuthrich, Philippe Mandin, Electrochimica Acta 54, 4031-4035 (2009).

8. Baoyang Jiang, Shuhuai Lan, Jun Ni, Zhaouyang Zhang, J. of Mat. Proc. Tech. 214, 892898 (2014) 